\title{
27 \\ Performance Prediction of P/M Electrodes in EDM Using Artificial Neural Network
}

\author{
P.K.Philip, Mathews P.Samuel, Thomas George, \\ G.Kuppuswamy
}

Department of Mechanical Engineering

Indian Institute of Technology, Madras 600 036, India

Tel: 2351365 Extn:3624, FAX: 9144 2350509/2352545

E-Mail: philip@acer.iitm.ernet.in

\begin{abstract}
Tool electrode is a vital factor in Electrical Discharge Machining (EDM). The non-availability of an ideal single tool material points to the Powder Metallurgy $(\mathrm{P} / \mathrm{M})$ process for combining the desirable properties of different tool materials. The properties of electrodes prepared by P/M route are determined by the powder characteristics as well as process variables. Due to the complex nature of electrical discharge machining, it is very difficult to determine the optimum conditions for improving machining performance. In this paper, a feed forward backpropagation neural network is used to correlate the process parameters with electrode performance. Experimental results have shown that the machining performance of EDM can be estimated and consequently improved upon by this method.
\end{abstract}

Keywords

EDM, P/M Electrodes, Performance indices ,Neural Network, Back Propagation, Machining estimation. 


\section{INTRODUCTION}

The erosive effect of electrical discharges on metals has been known for a long time, but the first step to use it for machining was made by Lazarenko in 1946 (1). Now it is a well developed technique for the manufacture of dies and moulds. Due to the technological developments in the past few decades, Electrical Discharge Machining (EDM) can provide an effective solution for machining hard conductive materials and reproducing complex shapes. Sophisticated computer control with fully automated work handling systems have enhanced the capability of EDM process (2). Even in the midst of all these technical excellence, selection of various input parameters for obtaining higher performance is not yet fully solved $(3,4)$.

Electrical discharge machining involves complicated phenomena such as spark initiation, dielectric breakdown, thermo-mechanical erosion of metal etc. (5). Due to the stochastic nature of EDM process, it is very difficult to map input factors to cutting performance using the conventional techniques. But neural networks are highly flexible modeling tools with an ability to learn the mapping between input and output variables (6-10). The advantage of neural network is utilised here to predict the machining performance of each electrode. A multi-layered, feed forward neural network with supervised learning has been used in the present work. The input-output data are normalised before the initiation of the neural network for better convergence and accuracy of the process. Since electrical discharge machining is basically a metal removal process, the amount of metal removed form the work piece in unit time and the electrode wear are the most important factors to be considered for evaluating the performance of a tool. For a comparative evaluation of such factors the conventional solid (forged) copper electrode is selected as a reference. Based on the performance of this conventional solid electrode. Powder Metallurgy ( $\mathrm{P} / \mathrm{M})$ electrodes can be evaluated as described in the following pages.

\section{POWDER METALLURGY ELECTRODES IN EDM}

EDM is based on the controlled erosion of materials by elelctric sparks between tool and work piece submerged in a bath of dielectric medium. The EDM process as it is understood today, can be divided into three sub divisions.

1. Application of adequate electrical energy

2. Dielectric breakdown and spark phenomena

3. Expulsion (erosion) of materials. 
Although this is a simple depiction of a complex process, it is sufficient to provide an overall perspective. Figure 1 shows the factors which affect the overall EDM performance. Among these factors, electrodes play a vital role in machining performance and accuracy $(11,12)$.

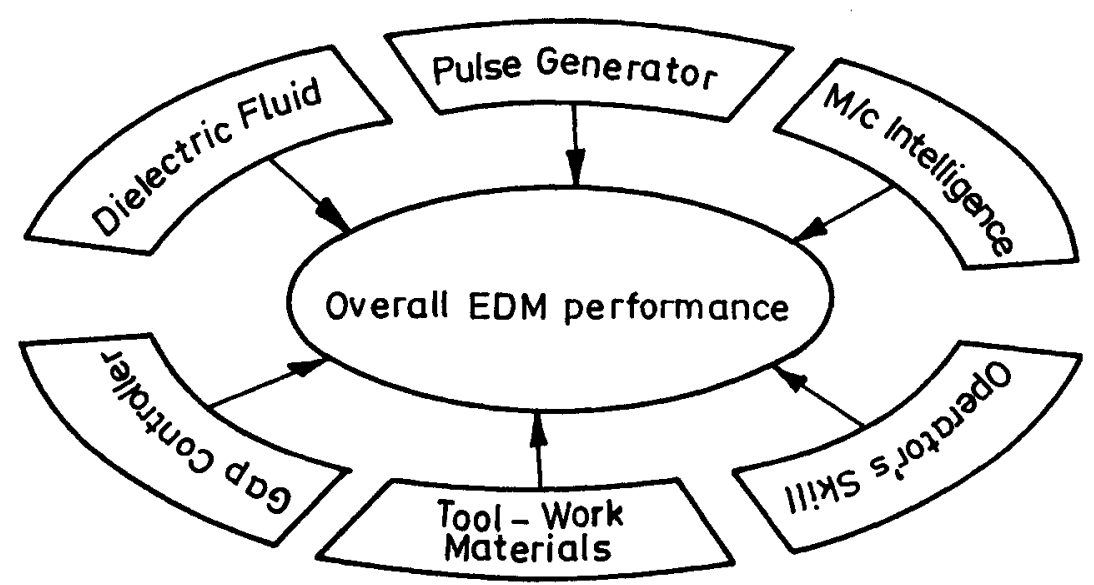

Figure 1 EDM Performance as a function of different parameters

The tool electrode material should not only be resistant to electric erosion, but also be capable of removing maximum material from the opposite electrode (work piece). Although an ideal single material with such a property does not exist, powder metallurgy ( $\mathrm{P} / \mathrm{M})$ provides a means of combining desirable characteristics of different materials. In this process compacting and sintering conditions as well as powder characteristics are decisive factors. Judicious selection of operating variables results in optimum electrode performance.

The results of EDM with P/M electrodes do not match completely with the conventional. These electrodes produce varying responses depending on the powder metallurgy conditions employed in their manufacture. In short, powder metallurgy process variables control the electrode properties and thereby dictate the performance of $\mathrm{P} / \mathrm{M}$ electrodes in EDM. In order to describe the performance behavior of $\mathrm{P} / \mathrm{M}$ electrodes, following performance factors are defined in this paper.

1. Machining Index (MI): It is the ratio of metal removed by a P/M electrode to that of by the conventional solid copper electrode of same dimensions operating under similar conditions. 
2. Electrode Performance Number (EPN). It is the ratio of the wear ratio of a $\mathrm{P} / \mathrm{M}$ electrode to that of a conventional solid copper electrode of same dimensions operating under similar conditions.

3. Material Removal Rate (MRR): It is the amount of work material removed by the electrode per unit time.

MI and EPN of solid copper electrode is fixed as 100 and based on this reference $\mathrm{P} / \mathrm{M}$ electrodes are graded. Negative value in any of these factors indicates metal deposition rather than metal removal.

\section{EXPERIMENTAL DETAILS}

Electrolytic copper ( $99.7 \%$ pure) powder was the starting material. A double cone blender was used for mixing the lubricant ( $1 \%$ zinc stearate by wt.) with the powder and the electrodes were prepared in a single acting press with die assembly. The electrodes prepared at different compacting pressures were sintered to different levels using a tubular furnace. The detailed specifications of compaction and sintering are given in table 1 .

\section{Table 1 Specification of Compaction and Sintering}

\section{COMPACTION}

Press capacity

Mode of compaction

Diameter of compact

Length of compact

Compaction range

\section{SINTERING}

Type of furnace

Protective atmosphere

Heating Rate

Pre-sintering Temp.

Max.Temperature

Sintering time
20 Ton

Single action

$14 \mathrm{~mm}$

$12 \mathrm{~mm}$

62 to $625 \mathrm{MPa}$

Performance evaluation of these P/M electrodes were carried out by Nassovia Krupp-31 discharge machine equipped with iso-frequent pulse generator. Kerosene was the dielectric medium and hardened steel $(53 \mathrm{HRC})$ was used as work piece for all experiments. Experiments were conducted with different settings of Pulse 
current and pulse frequency. The voltage and current pulses were recorded simultaneously using a dual trace oscilloscope connected to a digital plotter. Metal erosion was determined by weighing the electrodes before and after the experiments.

\section{NEURAL NETWORK MODELING}

Neural networks are based on the working pattern of brain cells or neurons and represent models different from those related to physical symbol systems such as expert systems where artificial intelligence(AI) features refer to performance paradigm of human reasoning. Neural systems are associated with what is called brain paradigm of AI methods. Typically the network consists of nodes and arcs, each of the nodes or circles modeling a very simplified neuron and arcs treated as connections. The nodes compute by responding to its inputs and the weights on the concerned arcs by the weighted sum rule.

Neuron is basic to the operation of neural networks by serving as an information processing unit. Fig. 2 illustrates the model of a typical neuron.

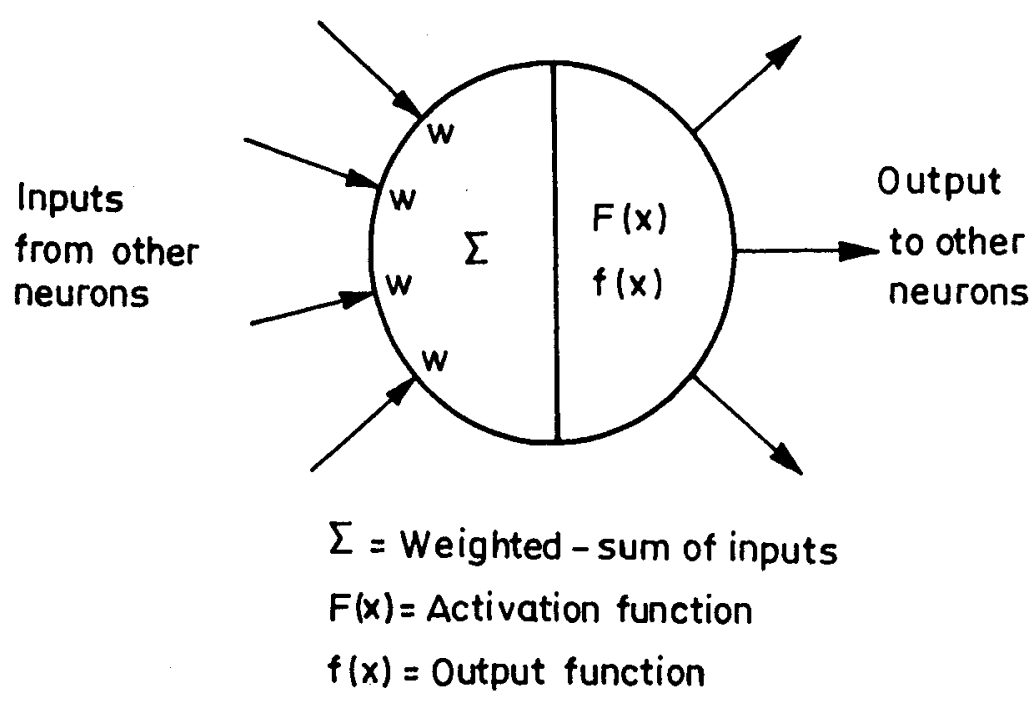

Figure 2 Working of Neuron model

The sum of weighted inputs from other neurons is processed based on specified threshold or activation and output functions to obtain the output to other neurons. The cells activation is determined by an activation function $F(x)$ specifying how the input is combined with the current activation value in order to produce the new activation value, or activation level. The final output of the cell is on computation 
by another function, the output function $f(x)$ on its activation level, typically by using a sigmoidal transfer function. This output value is further transmitted along the weighted outgoing connections as inputs to subsequent neurons.

Neural nets basically describe mathematical models of information processing and provide a method of representing relationships between input and output variables quite different from other computer methods with stored programs for functional models. The neural method had been chosen in the present study of performance of P/M electrodes in EDM since the problem is complex. The EDM output variables are not only dependent on pulse characteristics viz. frequency and current but also on electrode processing parameters, namely, powder size, compaction pressure and sintering temperature. The mathematical model formulation is thus difficult. Neural network model based on a typical sample data becomes a powerful simulation tool for further analysis regarding the influence of changes in parameters of the concerned variables on EDM performance indices, by providing reasonable output estimates.

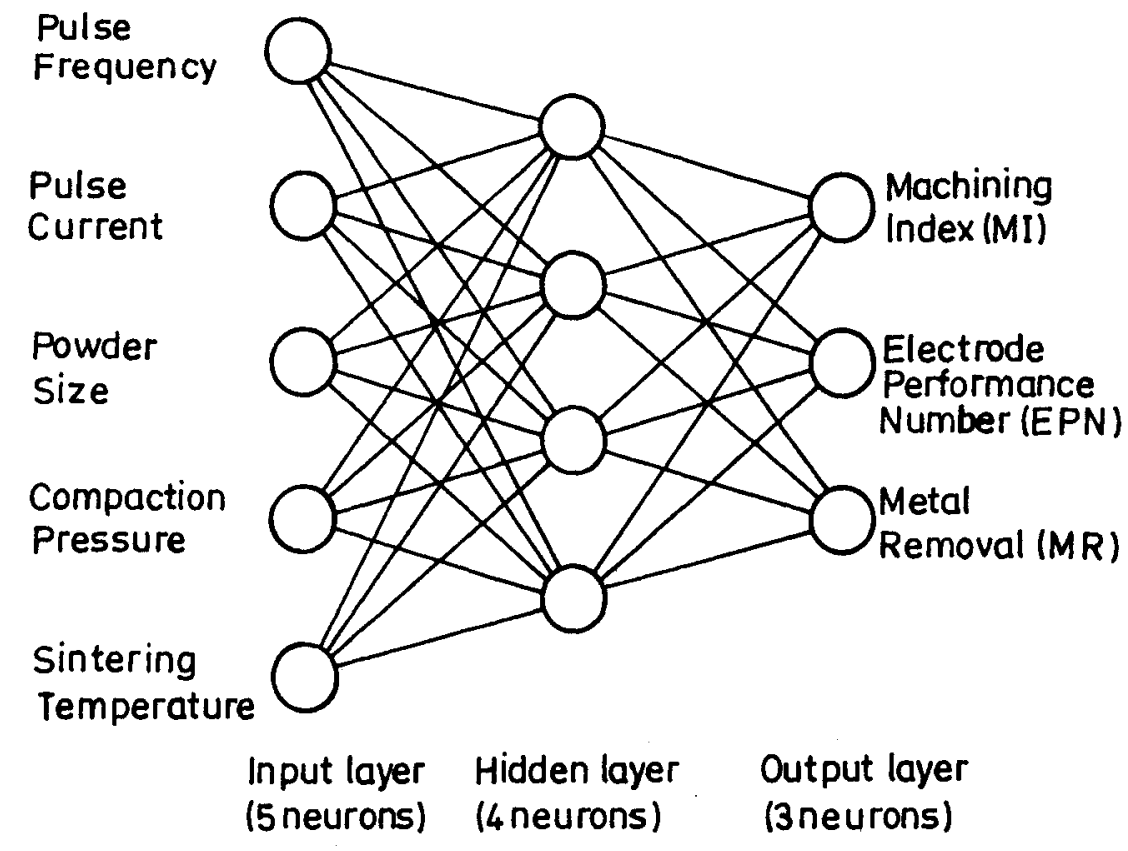

Figure 3 A multi-layered (5-4-3) back propagation neural network model

The feed-forward back propagation net is a popular architecture among different types of neural networks and finds applications in several areas in engineering. 
Fig. 3 represents a model having 5 neurons in the input layer, 4 neurons in the hidden layer and 3 neurons in the output layer. This network is used for analysis in the present study. Typically the network is trained for supervised learning with known input vector and output vector pairs to arrive at the arc weights for a prescribed error level, often requiring a large number of iterations or cycles of computation based on the back propagation algorithm. The backpropagation is a learning method that computes an error at each layer and propagates this error backward to determine appropriate improvements in weights.

For training the model, parameters for pulse frequency, pulse current, powder size, compaction pressure and sintering temperature have been used as inputs. Machining Index (MI) Electrode Performance Number (EPN) and Metal Removal (MR) are considered as outputs. A software written in $\mathrm{C}++$ for the back propagation method (7) was run on a $486 \mathrm{PC} / \mathrm{AT}$. Totally $15 \mathrm{~K}$ cycles of iterations were necessary to arrive at the cycle error of $7.78 \mathrm{E}-05$. This session was carried out in four stages by modifying the learning rate, momentum and noise factors used in the algorithm. The first phase had been for $3.5 \mathrm{~K}$ iterations at a learning rate of 0.5 , the second one for $1.5 \mathrm{~K}$ iterations at a learning rate of 0.1 and the last two for $10 \mathrm{~K}$ iterations at a learning rate of 0.01 . The error levels arrived at are $1.8 \mathrm{E}$ $04,1.28 \mathrm{E}-04,9.70 \mathrm{E}-05$, and finally $7.78 \mathrm{E}-05$ after the respective phases.

Table 2 represents the data used for training the network, and the final output values obtained.

Table 2 Data used for training and output results

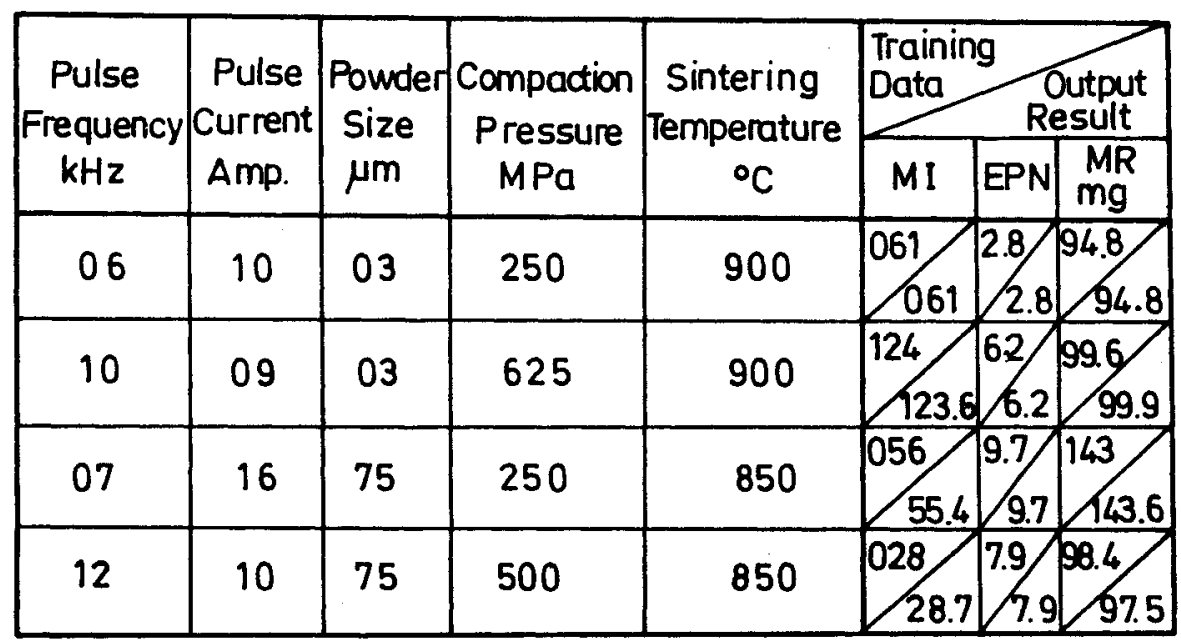




\section{SIMULATION RESULTS}

The neural network model developed is used as a simulation tool to evaluate the performance of P/M tool electrodes in EDM by estimating the MI, EPN and MR parameters. Table 3 shows the model output for a study on changes in powder size for a compaction pressure of $540 \mathrm{MPa}$, sintering temperature of $850^{\circ} \mathrm{C}$ at a pulse frequency of $8 \mathrm{KHz}$ and pulse current of $10 \mathrm{~A}$. The Table illustrates the use of the method to the manufacturing technologist. The model provides output estimates of the performance indices based on specified input parameters for further analysis. The model possesses potential for process monitoring, adaptive control as well as optimisation of electrode processing parameters. The results imply that the EDM performance is not only dependent on electrical pulse characateristics but also to a large extent on the electrode processing parameters. The model proves to be an appropriate tool for analysing the complex phenomena encountered in EDM involving a number of significant variables.

Table 3 Model-estimates at different levels of powder size

\begin{tabular}{|c|c|c|c|}
\hline $\begin{array}{c}\text { Powder } \\
\text { Size } \\
\mu \mathrm{m}\end{array}$ & MI & EPN & $\begin{array}{c}\text { MR } \\
\mathrm{mg}\end{array}$ \\
\hline 15 & 91.4 & 6.41 & 98.36 \\
30 & 66.9 & 6.83 & 96.99 \\
45 & 47.8 & 7.09 & 95.44 \\
65 & 35.6 & 7.36 & 94.31 \\
\hline
\end{tabular}

\section{CONCLUSIONS}

A multi-layered feed-forward back propagation neural network has been used to construct a model of Electrical Discharge Machining incorporating electrode manufacturing process parameters. The study illustrates that the network model can effectively map different process variables to machining performance of $P / M$ electrodes in terms of machining index, electrode performance number and metal removal and be used as a simulation tool for further analysis and optimisation. The results endorse the application of $\mathrm{P} / \mathrm{M}$ electrodes in EDM and the dependence of $P / M$ processing variables on its performance. 


\section{REFERENCES}

1. Lazarenko B.R.(1964) Electro spark machining of metals, Consultants Bureau, NY.

2. De Vries M.F, Duffie N.A, Kruth J, Dauw D.F, and Schumacher B (1990) Integration of EDM with CIM environment. Annals of the CIRP, Vol.39, pp $665-672$

3. Bayramoglu M and Dufil A.W (1994) Systematic investigation on the use of cylindrical tools for the reduction of 3D complex shapes on CNC EDM machines. Int.J.Mech.Tools Manuf.Vol.34,No.3.pp 327-339.

4. Rajurkar K P.(1990) Technology and research in EDM and ECM. ASME Fundamental Issue Mach. Vol.453, pp 309-336.

5. Erden E.(1983) Effect of materials on the mechanism of EDM. Trans of the ASME Vol.105, pp 132-138.

6. Nayes, J.L.(1992) Artificial Intelligence with common lisp fundamentals of symbolic and numerric processing, D.C. Heath and Co., MA, USA.

7. Rao V and Rao H, (1994) $\mathrm{C}++$ neural networks and fuzzy logic, $M \& T$ Publishing Inc. USA.

8. Lee B.Y, Tang Y.S.,Ma S.C.(1995) Modeling of the process damping force in chatter vibration, Int.J.Mach Tools.Manufact.Vol.35, No.7, pp 963-974.

9. Liao T.W. Chen L.J.(1994) A neural network approach for grinding process: Modeling and optimization, Int.J Mach Tools Manufact. Vol.34, No. 7, pp 919937.

10. Tang Y.S. Ma S.C.,Chung, I.K.(1995) Determination of optical cutting parameters in wire electrical discharge machining Int. J.Mach.Tools Manufact. Vol.46, No.12, pp 1693-1701.

11. Jeswani M.J. (1979) Dimensional analysis of tool wear in EDM, Int.J. of Wear, Vol.55, pp 153-161.

12. Meyrin S.A, Dauw D.F and Albert L (1992)About the evolution of wire tool performance in wire EDM, Annals of the CIRP, Vol.11/1, pp 221-225. 


\section{BIOGRAPHY}

Prof.P.K.PHILIP is Professor of Manufacturing Engineering, Department of Mechanical Engineering, Indian Institute of Technology, Madras from 1984. He was a doctoral scholar under the German Academic Exchange Programme (196870) and a Post-doctoral Fellow of the Alexander von Humboldt Foundation, Bonn (1975-77) both at the University of Stuttgart, Germany. He has published a large number of papers in his field of interest, which is material removal processes. He is a life fellow of the Institution of Engineers, India and a senior member of the SME from 1988 .

Mathews P.Samuel finished his M.S. research degree and is currently Scientist B at the Centre for Military Airworthiness and Certification, Bangalore 560035.

Thomas George was M.Tech. student at IIT Madras and is at present Lecturer in Mechanical Engineering at M.A.College of Engineering, Kothamangalam, Kerala 686666 .

Dr.G.Kuppuswamy is Associate Professor in Manufacturing Engineering at IIT Madras. 